\title{
ESTIMATIVA DA VELOCIDADE DE CISALHAMENTO EM DIFERENTES MODELOS E ESCALAS DE BIORREATORES PNEUMÁTICOS OPERADOS COM FLUIDOS NÃO- NEWTONIANOS
}

\author{
C.E. MENDES $^{1}$ e A.C. BADINO ${ }^{1}$ \\ ${ }^{1}$ Universidade Federal de São Carlos, Departamento de Engenharia Química \\ Email para contato: carol_engquimica@yahoo.com.br
}

\begin{abstract}
RESUMO - A velocidade de cisalhamento é um parâmetro de suma importância para o estudo do comportamento de reatores em bioprocessos, uma vez que pode gerar danos celulares, afetando adversamente o microrganismo e a eficiência do processo. No presente trabalho foi avaliada a velocidade média de cisalhamento $\left(\dot{\gamma}_{m}\right)$ em três biorreatores pneumáticos; coluna de bolhas (CB), airlift de cilindros concêntricos (ACC) e airlift split-cylinder (ASC), nas escalas de 5 e 10 L, para diferentes fluidos não-newtonianos (soluções de goma xantana-GX e carboximetilcelulose-CMC) e vazões de ar (1 a 5 vvm), obtida a partir de valores do coeficiente volumétrico de transferência de oxigênio $\left(\mathrm{k}_{\mathrm{L}} \mathrm{a}\right)$. Para o $\mathrm{k}_{\mathrm{L}} \mathrm{a}$, foram obtidos maiores valores no reator ACC de $10 \mathrm{~L}\left(\mathrm{k}_{\mathrm{L}} \mathrm{a}=0,056 \mathrm{~s}^{-1}\right)$. Com relação ao cisalhamento, foram observados maiores valores de $\dot{\gamma}_{m}$ para soluções de CMC menos viscosas em reator airlift de $10 \mathrm{~L}\left(\dot{\gamma}_{\mathrm{ACC}}=6,83 \times 10^{8} \mathrm{~s}^{-1}\right)$. Em geral, valores de $\dot{\gamma}_{m}$ reduziram com o aumento da vazão de ar.
\end{abstract}

\section{INTRODUÇÃO}

Uma vasta gama de modelos de reatores encontra-se disponível para utilização em bioprocessos, mas recentemente biorreatores pneumáticos têm sido considerados bem adaptados a processos biotecnológicos. Reatores pneumáticos estão substituindo reatores convencionais principalmente em processos aeróbios, devido a sua construção simples, baixo consumo energético, boas características de transferência de massa, calor e movimento e padrões de escoamento mais bem definidos, o que contribui adicionalmente para campos de cisalhamento homogêneos ao longo do reator (Bannari et al., 2011).

A estimativa da velocidade de cisalhamento média $\left(\dot{\gamma}_{\mathrm{m}}\right)$ em um processo bioquímico tem grande importância, pois dependendo da intensidade, um campo de cisalhamento pode ser estimulante, inibidor ou totalmente destrutivo, afetando diferentes tipos de células, principalmente microrganismos filamentosos. Tais campos de cisalhamento mesmo em baixos níveis podem afetar a morfologia celular (Jonczyk et al., 2013), formação de pellets e biofilmes (Cui et al., 1998) e instabilidade mecânica de biocatalisadores imobilizados (Martins et al., 1997). Tais influências refletem em aspectos industrialmente importantes como a produtividade, produtos gerados e suas características (Chisti, 2009). 
Ademais, o conhecimento da velocidade de cisalhamento média permite correlacionar dados de hidrodinâmica e transferência de massa em sistemas não-newtonianos. A velocidade de transferência de massa depende fortemente da hidrodinâmica em regiões próximas da interface gás-líquido e, portanto, o $\dot{\gamma}_{\mathrm{m}}$ afeta a transferência de massa (Contreras et al., 1999). Portanto, levando em consideração que o cisalhamento pode afetar adversamente às células e contribuir para transferência de massa, o estudo da velocidade de cisalhamento é essencial para o projeto e otimização de biorreatores.

Algumas correlações encontram-se disponíveis na literatura para previsão de $\dot{\gamma}_{\mathrm{m}}$, em sua maioria, disponíveis apenas para reatores coluna de bolhas. A correlação de Nishikawa et al. (1977), descrita de forma generalizada pela Equação 1, tem sido amplamente utilizada na literatura para os sistemas não-newtonianos em reatores pneumáticos, com parâmetro $\alpha$ variando de 1000 a $5000 \mathrm{~m}^{-1}$ (Nishikawa et al., 1977; Schumpe e Deckwer, 1987).

$$
\dot{\gamma}_{\mathrm{m}}=\alpha \cdot U_{G R}^{\beta}
$$

Embora esteja claro que a relação entre $\dot{\gamma}_{\mathrm{m}}$ e transferência de massa em reatores pneumáticos deva existir, poucos são os estudos que abordam este ponto. Merchuk e Ben-Zvi (1992) verificaram que existe uma relação simples entre o coeficiente volumétrico de transferência de massa $\left(\mathrm{k}_{\mathrm{L}} \mathrm{a}\right)$ e $\dot{\gamma}_{\mathrm{m}}$ para diferentes fluidos em reator $\mathrm{CB}$, entretanto, somente em 2008, Cerri e colaboradores reportaram a obtenção de $\dot{\gamma}_{m}$ utilizando como parâmetro característico o $\mathrm{k}_{\mathrm{L}} \mathrm{a}$, fazendo uma analogia entre fluidos newtonianos e não-newtonianos (Equação 2), analogia esta preconizada por Metzner e Otto (1957).

$$
\dot{\gamma}_{\mathrm{m}}=\left(\frac{1}{\mathrm{~K}}\right)^{1 / \mathrm{n}-1} \cdot\left(\frac{\mathrm{k}_{\mathrm{L}} \mathrm{a}}{\beta \cdot \mathrm{U}_{\mathrm{GR}}^{\varphi}}\right)^{1 / \theta} \cdot(\mathrm{n}-1)
$$

Desta forma, devido a informações limitadas quanto ao cisalhamento em reatores pneumáticos, principalmente reatores airlift, este trabalho teve como objetivo prever a velocidade média de cisalhamento em diferentes modelos e escalas de reatores pneumáticos, com o intuito de melhor compreender os fenômenos físicos que influenciam a eficiência de bioprocessos realizados nesses reatores.

\section{MATERIAL E MÉTODOS}

\subsection{EQUIPAMENTOS}

Os experimentos foram realizados em três tipos distintos de reatores pneumáticos: um coluna de bolhas (CB) e dois modelos de airlift de circulação interna, cilindros concêntricos (ACC) e split-cylinder (ASC). Cada reator foi avaliado em duas escalas: 5 e $10 \mathrm{~L}$ de volume útil, sendo as escalas geometricamente semelhantes.

Os equipamentos apresentam cilindro externo confeccionado em vidro borosilicato transparente acoplado a uma base e tampa superior de aço inoxidável $316 \mathrm{~L}$, sendo os reatores de $5 \mathrm{~L}$ e $10 \mathrm{~L}$, com $125 \mathrm{~mm}$ e $160 \mathrm{~mm}$ de diâmetro interno (Di), respectivamente. Os reatores ACC possuem um cilindro interno de aço inoxidável 316L (5 L: Di=75 mm e altura $(\mathrm{h})=350$ $\mathrm{mm}$; $10 \mathrm{~L}: \mathrm{Di}=95 \mathrm{~mm} \mathrm{e} \mathrm{h}=450 \mathrm{~mm}$ ), enquanto os reatores ASC possuem placa interna 


\section{9 a 22 de outubro de 2014 \\ Florianópolis/SC}

igualmente de aço (quociente da área de descida pela área de subida de 1,38 para ambos os tamanhos). Nos modelos de reatores CB e ACC utilizou-se aspersor do tipo cruzeta, confeccionado em aço inoxidável $316 \mathrm{~L}$, com orifícios de $0,5 \mathrm{~mm}$ de diâmetro e espaçamento entre si de $5 \mathrm{~mm}$ ao longo do comprimento das hastes do aspersor. Para o reator ASC utilizouse um aspersor tipo espinha-de-peixe.

\subsection{VELOCIDADE MÉDIA DE CISALHAMENTO $\left(\dot{\gamma}_{\mathrm{m}}\right)$}

Para previsão do $\dot{\gamma}_{\mathrm{m}}$ utilizando fluidos não-newtonianos, utilizou-se o método descrito por Cerri et al. (2008), onde presume-se que para um dado coeficiente volumétrico de transferência de oxigênio $\left(\mathrm{k}_{\mathrm{L}} \mathrm{a}\right)$, fluidos newtonianos e não-newtonianos apresentam a mesma viscosidade, ( $\mu$ para fluidos newtonianos e $\mu_{\mathrm{ap}}$ para fluidos não-newtonianos), sob mesmas condições de operação. Desta forma, água e soluções de glicerol (GS) foram utilizados como fluidos newtonianos e soluções de goma-xantana (GX) e carboxi-metil-celulose (CMC) foram utilizados como fluidos não-newtonianos, sendo a temperatura das soluções mantida à $30^{\circ} \mathrm{C}$.

A viscosidade dinâmica das soluções de fluidos newtonianos, assim como os parâmetros reológicos do modelo da lei de potência das soluções de fluidos não-newtonianos foram determinados com auxílio de um reômetro digital de cilindros concêntricos (modelo LV-DVIII+, Brookfield Engineering Laboratories) à $30^{\circ} \mathrm{C}$. Tais propriedades reológicas podem ser visualizadas na Tabela 1. Como fase gasosa utilizou-se ar, o qual foi injetado no reator com auxílio de um fluxômetro de massa (modelo GFC371, AALBORG) em cinco vazões que variaram uniformemente de 1 a 5 vvm.

Tabela 1 - Propriedades físicas dos fluidos utilizados como fase líquida

\begin{tabular}{ccccccc}
\hline Solução & $\mathrm{m} \cdot \mathrm{v}^{-1}(\%)$ & $\mathrm{K} \times 10^{3}\left(\mathrm{~Pa}_{\mathrm{s}} \mathrm{s}^{\mathrm{n}}\right)$ & $\mathrm{n}(-)$ & Solução & $\mathrm{v}^{-1}(\%)$ & $\mu \times 10^{3}(\mathrm{~Pa} . \mathrm{s})$ \\
\hline CMC15 & 0,15 & 63,2 & 0,79 & Água & 0 & 0,84 \\
CMC25 & 0,25 & 106,8 & 0,77 & GS10 & 63 & 10,0 \\
CMC35 & 0,35 & 170,2 & 0,75 & GS20 & 75 & 20,0 \\
CMC45 & 0,45 & 287,4 & 0,73 & GS25 & 79 & 25,0 \\
GX15 & 0,15 & 274,5 & 0,43 & GS30 & 82 & 30,0 \\
GX20 & 0,20 & 601,0 & 0,36 & & & \\
GX25 & 0,25 & 1044,0 & 0,29 & & & \\
GX30 & 0,30 & 1368,0 & 0,25 & & & \\
\hline
\end{tabular}

Pela metodologia de Cerri et al. (2008), os dados experimentais de $\mathrm{k}_{\mathrm{L}} \mathrm{a}$ dos fluidos newtonianos, para diferentes viscosidades e velocidades superficiais do gás na região de subida $\left(\mathrm{U}_{\mathrm{GR}}\right)$ podem ser correlacionados pela Equação 3 obtendo-se os coeficientes $\beta, \theta$ e $\varphi$.

$$
\mathrm{k}_{\mathrm{L}} \mathrm{a}=\beta \cdot \mathrm{U}_{\mathrm{GR}}^{\varphi} \cdot \mu^{\theta}
$$

Por fim, $\dot{\gamma}_{m}$ para fluidos não-newtonianos pode ser obtida pela Equação 2, utilizando-se os parâmetros $\beta, \theta$ e $\varphi$ obtidos para fluidos newtonianos e valores de $\mathrm{k}_{\mathrm{L}} \mathrm{a}, \mathrm{K}$ e $\mathrm{n}$ obtidos para os fluidos não-newtonianos. 


\subsection{COEFICIENTE VOLUMÉTRICO DE TRANSFERÊNCIA DE OXIGÊNIO $\left(k_{L} \mathbf{a}\right)$}

Para obtenção do $\mathrm{k}_{\mathrm{L}} \mathrm{a}$ nos reatores em cada condição operacional, utilizou-se o método de degrau de pressão (Linek et al., 1993). A concentração de oxigênio dissolvido foi medida empregando um analisador de oxigênio dissolvido (modelo O2 4100e, Mettler Toledo) conectado a um sistema de aquisição de dados. Para medição da pressão, utilizou-se um medidor de pressão eletrônico (modelo SC990, T\&S Equipamentos eletrônicos). O degrau de pressão foi de aproximadamente $15 \mathrm{kPa}$ sendo o valor de $\mathrm{k}_{\mathrm{L}}$ a determinado pelo ajuste dos dados à Equação 4.

$$
C_{e}=C_{e 0} \cdot e^{-k_{e} \cdot\left(t-t_{0}\right)}+C_{S} \cdot\left(1-e^{-k_{e} \cdot\left(t-t_{0}\right)}\right)+\frac{k_{e} \cdot\left(C_{S}-C_{e 0}\right)}{k_{e}-k_{L} a} \cdot\left(e^{-k_{e} \cdot\left(t-t_{0}\right)}-e^{-k_{L} a \cdot\left(t-t_{0}\right)}\right)
$$

onde, $\mathrm{C}_{\mathrm{e}}$ e $\mathrm{C}_{\mathrm{e} 0}$ são as concentrações nos instantes $\mathrm{t}$ qualquer e $\mathrm{t}_{0}$ (tempo inicial) respectivamente, $\mathrm{C}_{\mathrm{s}}$ é a concentração de saturação de oxigênio no meio líquido e $\mathrm{k}_{\mathrm{e}}$ é a constante de atraso do eletrodo, determinada experimentalmente como sendo $0,114 \mathrm{~s}^{-1}$. Os dados de $\mathrm{k}_{\mathrm{L}}$ a foram obtidos em duplicata, respeitando um limite máximo de desvio de $3 \%$ entre as repetições. Para o ajuste dos dados às equações, utilizou-se o conjunto completo dos dados, sendo todos os ajustes realizados no software Origin 7.

\subsection{RESULTADOS E DISCUSSÃO}

Os valores de $\mathrm{k}_{\mathrm{L}} \mathrm{a}$ para fluidos newtonianos nas diferentes condições avaliadas ( $\phi_{\mathrm{ar}}$ de 1 a $5 \mathrm{vvm} ; \mu$ de 0,00084 a 0,030 Pa.s) foram superiores para reatores airlift, em especial para ACC de $10 \mathrm{~L}\left(0,002344 \leq \mathrm{k}_{\mathrm{L}} \mathrm{a}_{\mathrm{ACC} 10} \leq 0,07716 \mathrm{~s}^{-1}\right)$. Em todos os experimentos observou-se um comportamento diretamente proporcional do $\mathrm{k}_{\mathrm{L}}$ a com relação ao $\phi_{\mathrm{ar}}$ e volume do reator e inversamente proporcional a $\mu$ do líquido (fato que pode ser observado pelos coeficientes $\varphi \mathrm{e}$ $\theta$ apresentados na Tabela 2). Os menores valores desta variável foram observados para o reator CB de $5 \mathrm{~L}\left(0,001793 \leq \mathrm{k}_{\mathrm{L}} \mathrm{a}_{\mathrm{CB} 5} \leq 0,03975 \mathrm{~s}^{-1}\right)$.

$\mathrm{O}$ conjunto de dados formado por 25 experimentos de $\mathrm{k}_{\mathrm{L}}$ a realizados para cada reator foi utilizado para o ajuste da Equação 3, sendo os parâmetros $\beta, \varphi$ e $\theta$ obtidos para os diferentes modelos e escalas de reatores, apresentados na Tabela 2.

Tabela 2 - Parâmetros obtidos para correlação de $\mathrm{k}_{\mathrm{L}}$ a a partir da Equação 3, para fluidos newtonianos nos três modelos de biorreatores de 5 e $10 \mathrm{~L}$

\begin{tabular}{cccccc}
\hline \multirow{2}{*}{ Volume } & Reator & \multicolumn{3}{c}{ Parâmetros } & \multirow{2}{*}{$\mathrm{R}^{2}$} \\
\cline { 3 - 5 } & & $\beta\left(\times 10^{3}\right)$ & $\varphi$ & $\theta$ & \\
\hline \multirow{3}{*}{$5 \mathrm{~L}$} & ACC & $1,37 \pm 0,27$ & $0,586 \pm 0,035$ & $-0,727 \pm 0,024$ & 0,99 \\
& ASC & $1,32 \pm 0,30$ & $0,492 \pm 0,044$ & $-0,674 \pm 0,027$ & 0,99 \\
& CB & $2,27 \pm 0,37$ & $0,487 \pm 0,030$ & $-0,642 \pm 0,017$ & 0,99 \\
\hline \multirow{3}{*}{$10 \mathrm{~L}$} & ACC & $1,59 \pm 0,26$ & $0,701 \pm 0,035$ & $-0,712 \pm 0,021$ & 0,99 \\
& ASC & $1,52 \pm 0,24$ & $0,638 \pm 0,033$ & $-0,703 \pm 0,021$ & 0,99 \\
& CB & $3,05 \pm 0,47$ & $0,691 \pm 0,031$ & $-0,699 \pm 0,018$ & 0,99 \\
\hline
\end{tabular}


A Figura 1 expressa os valores de $\mathrm{k}_{\mathrm{L}} \mathrm{a}$ calculados com auxílio da Equação 3 e Tabela 2, em comparação aos valores de $\mathrm{k}_{\mathrm{L}} \mathrm{a}$ experimentais para os três modelos de biorreatores nas duas escalas. Para todos os biorreatores, ótimas correlações foram obtidas, as quais são capazes de prever $99 \%$ dos dados experimentais (Tabela 2), com erro inferior a $15 \%$ em comparação aos dados experimentais (Figura 1).

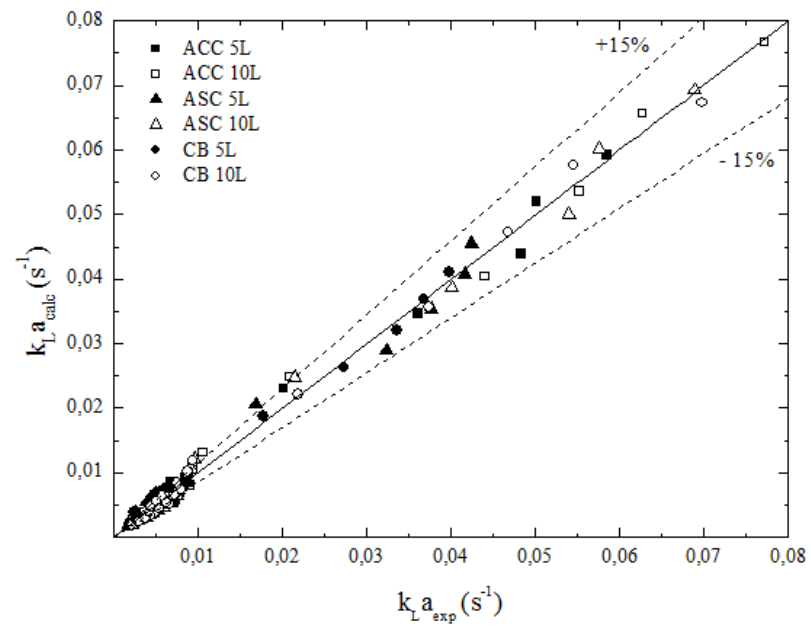

Figura 1 - Comparação entre valores calculados (Equação 3) e experimentais de $\mathrm{k}_{\mathrm{L}} \mathrm{a}$, para fluidos newtonianos nos reatores $\mathrm{CB}, \mathrm{ACC}$ e ASC de 5 e $10 \mathrm{~L}$.

Um comportamento semelhante aos fluidos newtonianos foi observado para fluidos não-newtonianos, onde o $\mathrm{k}_{\mathrm{L}} \mathrm{a}$ aumentou com $\phi_{\mathrm{ar}}$ e volume do reator e diminuiu com o índice de consistência do fluido (K), como pode ser visualizado na Figura 2a, Figura 3a e Figura 4a. De forma análoga aos fluidos newtonianos, o reator ACC de $10 \mathrm{~L}$ foi o mais eficiente na transferência de oxigênio em fluidos não-newtonianos $\left(\mathrm{k}_{\mathrm{L}} \mathrm{a}=0,056 \mathrm{~s}^{-1}\right.$ em CMC15 e 5 vvm). Em geral, as soluções de CMC apresentaram maiores valores de $\mathrm{k}_{\mathrm{L}} \mathrm{a}$, entretanto, para um índice de consistência semelhante $\left(\mathrm{K} \cong 0,30 \mathrm{~Pa} \cdot \mathrm{s}^{\mathrm{n}}\right)$, a solução de $\mathrm{GX}$ que possui índice de escoamento menor do que $0,5(n=0,43)$ apresentou maiores valores de $k_{L} a\left(0,0139 \leq k_{L} a_{A C C 10}\right.$ $\left.\leq 0,03986 \mathrm{~s}^{-1}\right)$ em comparação a solução de CMC (n=0,73;0,006301 $\leq \mathrm{k}_{\mathrm{L}} \mathrm{a}_{\mathrm{ACC} 10} \leq 0,01707 \mathrm{~s}^{-}$ ${ }^{1}$ ). Com uma observação simples da combinação entre a Lei de Newton da viscosidade e a Lei da Potência que descreve a reologia de fluidos pseudoplásticos (Equação 5), observa-se que para um mesmo valor de $\mathrm{K}$, soluções de menor valor de $\mathrm{n}$ apresentam uma menor viscosidade aparente, fato que justifica o resultado obtido.

$$
\mu_{\mathrm{ap}}=\frac{\tau}{\dot{\gamma}}=\mathrm{K} \cdot \dot{\gamma}^{\mathrm{n}-1}
$$

Pela análise das Figuras 2b, $3 b$ e 4b, observa-se que os valores máximos de velocidade média de cisalhamento foram obtidos para reatores airlift do tipo ACC de $10 \mathrm{~L}$ $\left(\dot{\gamma}_{m \mathrm{ACC} 10}=6,83 \times 10^{8} \mathrm{~s}^{-1} ; \dot{\gamma}_{m \mathrm{ASC} 10}=5,37 \times 10^{8} \mathrm{~s}^{-1}, \mathrm{CMC} 15\right.$ e $\left.2 \mathrm{vvm}\right)$. As maiores vazões de ar em geral, geraram maior cisalhamento dos fluidos e ainda, com relação à reologia, as figuras permitem concluir que o $\mathrm{K}$ do fluido tem relação inversamente proporcional ao $\dot{\gamma}_{m}$. Ainda, com relação à reologia, observa-se que para fluidos com $\mathrm{K}$ semelhantes, o índice de escoamento (n) afeta positivamente o $\dot{\gamma}_{m}$. 
Nos reatores ACC, o $\dot{\gamma}_{m}$ apresentou um comportamento similar para os diferentes tipos de fluidos utilizados. Em geral, observa-se um decréscimo do $\dot{\gamma}_{m}$ com o aumento do $\phi_{\text {ar }}$ Para os menores índices de consistência de ambos os fluidos $\left(\mathrm{K}_{\mathrm{CMC}}=0,063\right.$ e 0,107 Pa.s ${ }^{\mathrm{n}}$; $\mathrm{K}_{\mathrm{GX}}=0,274$ e 0,600 Pa.s ${ }^{\mathrm{n}}$ ), ocorre um pico de cisalhamento em geral, em 1 e $2 \mathrm{vvm}$, com exceção das soluções de CMC no reator de $5 \mathrm{~L}$, no qual o valor máximo de cisalhamento ocorre em $3 \operatorname{vvm}\left(\dot{\gamma}_{m}=3,03 \times 10^{8} \mathrm{~s}^{-1}\right.$ em K=0,063 Pa.s ${ }^{\mathrm{n}}$ e $\dot{\gamma}_{m}=1,41 \times 10^{8} \mathrm{~s}^{-1}$ em K=0,107 Pa.s $\left.{ }^{\mathrm{n}}\right)$. As soluções mais concentradas de CMC e GX (K=0,287 e 1,368 Pa.s ${ }^{\mathrm{n}}$, respectivamente) apresentaram cisalhamento praticamente constante em todas as vazões, entretanto com um leve acréscimo nas maiores vazões (4 e $5 \mathrm{vvm}$ ). Resultados semelhantes foram obtidos por Cerri et al. (2008) em reator ACC de $5 \mathrm{~L}$, onde o $\dot{\gamma}_{\mathrm{m}}$ apresentou valores máximos em geral nas vazões entre 1 e 2 vvm para soluções de GX e decrescendo nas vazões subsequentes.

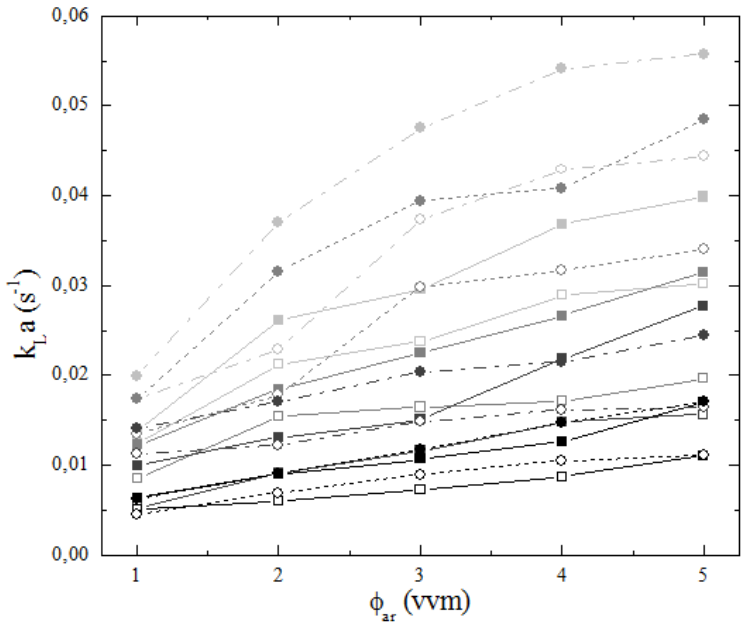

(a)

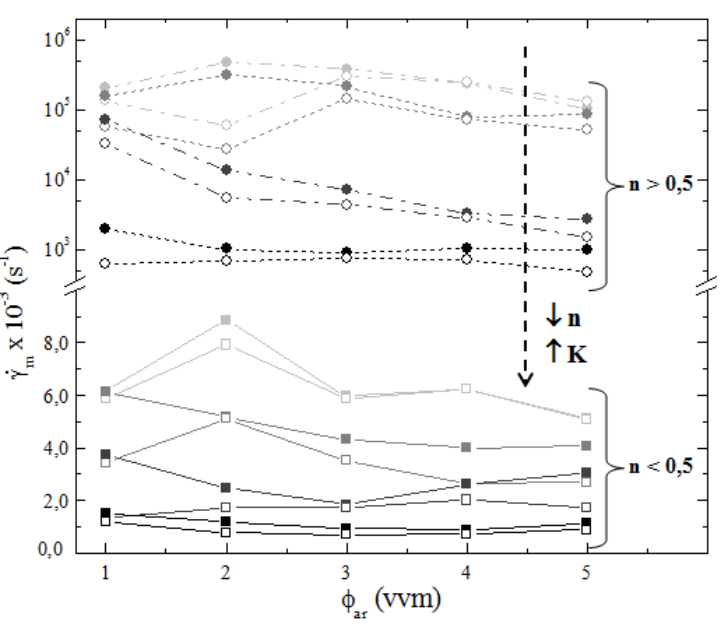

(b)

Figura 2 - a) Coeficiente volumétrico de transferência de oxigênio $\left(k_{\mathrm{L}} \mathrm{a}\right)$ em função da vazão de $\left.\operatorname{ar}\left(\phi_{\mathrm{ar}}\right) ; \mathrm{b}\right)$ velocidade de cisalhamento média $\left(\dot{\gamma}_{\mathrm{m}}\right)$ em função da vazão de ar $\left(\phi_{\mathrm{ar}}\right)$, para fluidos não-newtonianos nos reatores ACC de 5 e 10L. Legenda: $-=-10 \mathrm{~L}-\mathrm{GX} 15-\square-5 \mathrm{~L}-\mathrm{GX} 15$ $-\square-10 \mathrm{~L}-\mathrm{GX} 20-\square-5 \mathrm{~L}-\mathrm{GX} 20-\square-10 \mathrm{~L}-\mathrm{GX} 25-\square-5 \mathrm{~L}-\mathrm{GX} 25-\square-10 \mathrm{~L}-\mathrm{GX} 30-\square-5 \mathrm{~L}-\mathrm{GX} 30-\bullet-10 \mathrm{~L}-$ CMC15- - 5L-CMC15 ……10L-CMC25 … 5L-CMC25 - *- 10L-CMC35 - o- 5L-CMC35 …… 10LCMC45 …ㄴ..5L-CMC45.

Nos reatores ASC de 5 e $10 \mathrm{~L}$ de volume útil, observou-se um comportamento semelhante do $\mathrm{k}_{\mathrm{L}} \mathrm{a}$ com relação as variáveis avaliadas $\left(\phi_{\mathrm{ar}}\right.$ e $\mu$ ), conforme Figura $3 \mathrm{a}$. Os valores de $\mathrm{k}_{\mathrm{L}}$ a para este modelo de biorreator foram pouco inferiores aos obtidos para ACC mas, de forma análoga com o reator de $10 \mathrm{~L}$ sendo o mais eficiente na transferência de oxigênio, o qual apresentou $\mathrm{k}_{\mathrm{L}}$ a variando de 0,00588 a $0,05679 \mathrm{~s}^{-1}$ com $10 \mathrm{~L}$ de volume e de 0,00476 a $0,03691 \mathrm{~s}^{-1}$ para $5 \mathrm{~L}$, ambos para CMC15. De maneira geral, o comportamento do $\dot{\gamma}_{m}$ nos reatores ASC foi semelhante ao ACC e de forma análoga a variável $\mathrm{k}_{\mathrm{L}} \mathrm{a}$, somente apresentou valores pouco inferiores $\left(\dot{\gamma}_{m}=4,38 \times 10^{8} \mathrm{~s}^{-1}\right.$ e $\dot{\gamma}_{m}=5,37 \times 10^{8} \mathrm{~s}^{-1}$, para CMC15, $2 \mathrm{vvm}$ em reatores de 5 e $10 \mathrm{~L}$, respectivamente).

Resultados semelhantes aos reatores airlift foram observados para $\mathrm{CB}$, entretanto, tanto os valores de $\mathrm{k}_{\mathrm{L}}$ a quanto de $\dot{\gamma}_{m}$ para este modelo de biorreator são consideravelmente inferiores aos demais. Conforme a Figura $4 \mathrm{a}$, o $\mathrm{k}_{\mathrm{L}} \mathrm{a}$ para este modelo variou de 0,00508 a $0,03780 \mathrm{~s}^{-1}$ para o reator de $10 \mathrm{~L}$ e de 0,004199 a $0,02318 \mathrm{~s}^{-1}$ para $5 \mathrm{~L}$, com solução de 
CMC15. Tal solução também gerou maior cisalhamento neste reator, atingindo $\dot{\gamma}_{m}=1,83 \times 10^{8}$ $\mathrm{s}^{-1}$ para $10 \mathrm{~L}$ de volume útil e $\dot{\gamma}_{m}=1,31 \times 10^{8} \mathrm{~s}^{-1}$ para $5 \mathrm{~L}$, em vazão de $2 \mathrm{vvm}$.

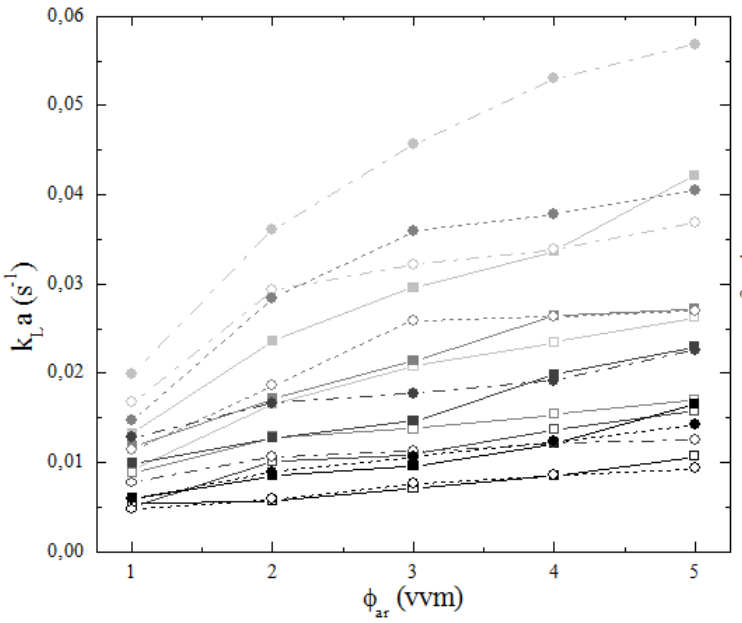

(a)

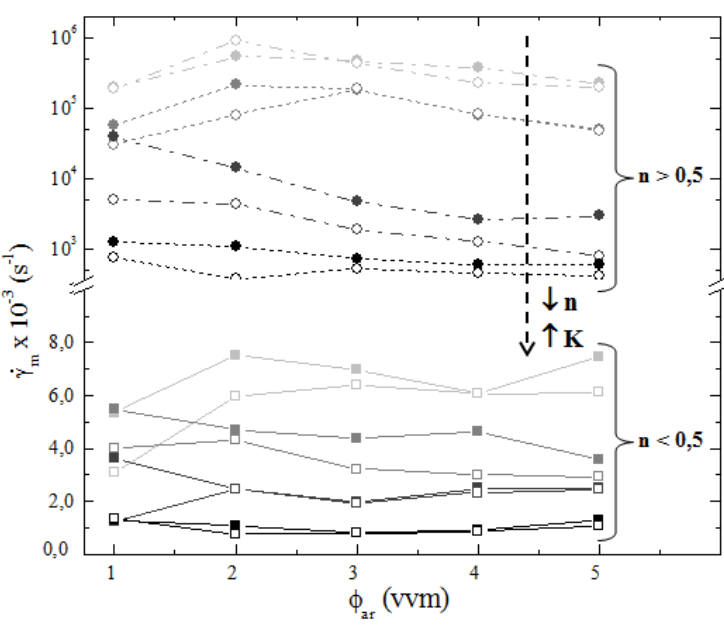

(b)

Figura 3 - a) Coeficiente volumétrico de transferência de oxigênio $\left(\mathrm{k}_{\mathrm{L}} \mathrm{a}\right)$ em função da vazão de $\left.\operatorname{ar}\left(\phi_{\mathrm{ar}}\right) ; \mathrm{b}\right)$ velocidade de cisalhamento média $\left(\dot{\gamma}_{\mathrm{m}}\right)$ em função da vazão de ar $\left(\phi_{\mathrm{ar}}\right)$, para fluidos não-newtonianos nos reatores ASC de 5 e 10L. Legenda: -"-10L-GX15 - - 5L-GX15

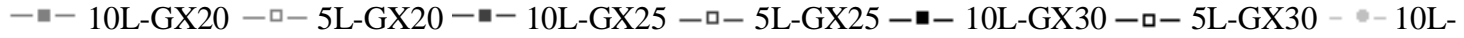
CMC15- - 5L-CMC15 ……10L-CMC25 …5L-CMC25 - *-10L-CMC35 - o- 5L-CMC35 … $\bullet$ 10LCMC45 … 5 L-CMC45.

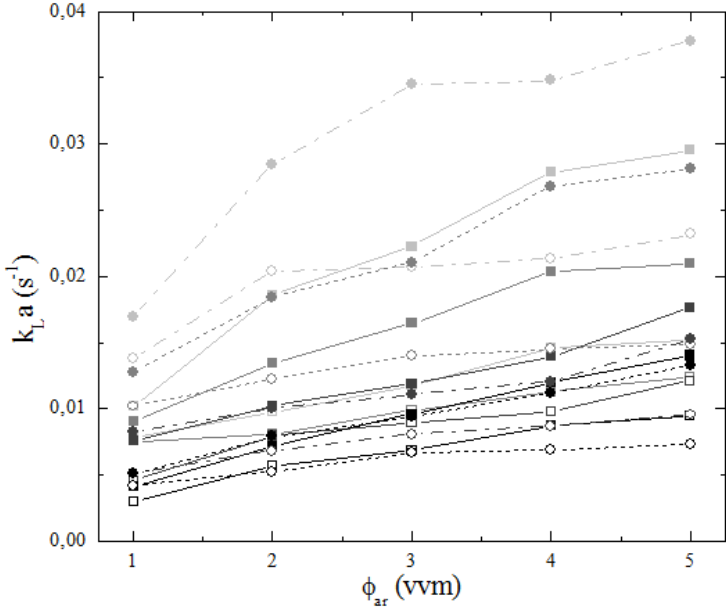

(a)

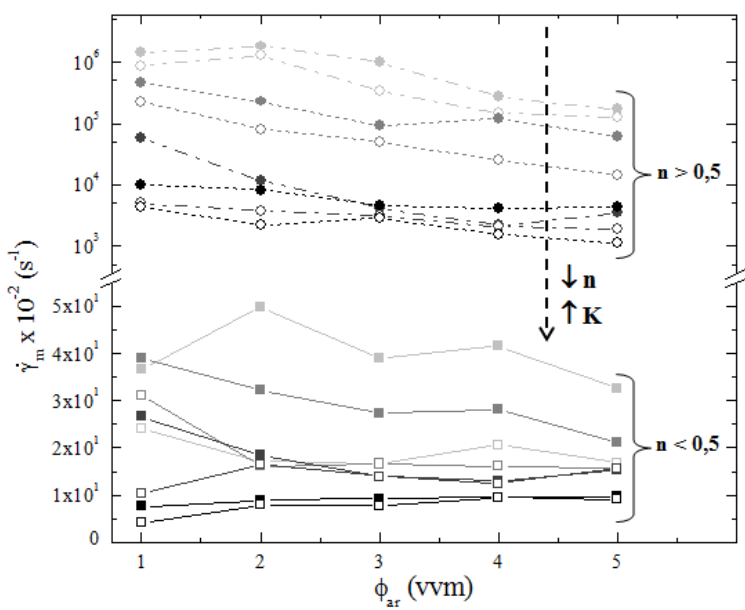

(b)

Figura 4 - a) Coeficiente volumétrico de transferência de oxigênio $\left(k_{\mathrm{L}} \mathrm{a}\right)$ em função da vazão de $\left.\operatorname{ar}\left(\phi_{\mathrm{ar}}\right) ; \mathrm{b}\right)$ velocidade de cisalhamento média $\left(\dot{\gamma}_{\mathrm{m}}\right)$ em função da vazão de ar $\left(\phi_{\mathrm{ar}}\right)$, para fluidos não-newtonianos nos reatores CB de 5 e 10L. Legenda: $-=-10 \mathrm{~L}-\mathrm{GX} 15-\square-5 \mathrm{~L}-\mathrm{GX} 15$ - $-10 \mathrm{~L}-\mathrm{GX} 20-\square-5 \mathrm{~L}-\mathrm{GX} 20-\square-10 \mathrm{~L}-\mathrm{GX} 25-\square-5 \mathrm{~L}-\mathrm{GX} 25-10 \mathrm{~L}-\mathrm{GX} 30-\square-5 \mathrm{~L}-\mathrm{GX} 30-10 \mathrm{~L}-$ CMC15- - 5L-CMC15 ……10L-CMC25 … 5L-CMC25 - *10L-CMC35 - o- 5L-CMC35 … $\bullet$ 10LCMC45 ……5L-CMC45.

\section{AGRADECIMENTOS}


Os autores agradecem a FAPESP pelo apoio financeiro (Proc. 2012/17756-8 e 2011/23807-1).

\section{REFERÊNCIAS}

BANNARI, R.; BANNARI, A.; SELMA, B.; PROULX, P. Mass transfer and shear in an airlift bioreactor: Using a mathematical model to improve reactor design and performance. Chem. Eng. Sci., v. 66, p. 2057-2067, 2011.

CERRI, M. O.; FUTIWAKI, L.; JESUS, C. D. F.; CRUZ, A. J. G.; BADINO, A. C. Average shear rate for non-Newtonian fluids in a concentric-tube airlift bioreactor. Biochem. Eng. J., v. 39, p. 51-57, 2008.

CHISTI, Y. Shear sensitivity. In: FLICKINGER, M. C. (Ed.). Encyclopedia of Industrial Biotechnology: Bioprocess, bioseparation, and cell technology. New York: John Wiley and Sons, 2009.

CONTRERAS, A.; GARCÍA, F.; MOLINA, E.; MERCHUK, J. C. Influence of sparger on energy dissipation, shear rate, and mass transfer to sea water in a concentric-tube airlift bioreactor. Enzyme Microb. Technol., v. 25, p. 820-830, 2009.

CUI, Y. Q.; VAN DER LANS, R. G. J. M.; LUYBEN, K. C. A. M. Effects of dissolved oxygen tension and mechanical forces on fungal morphology in submerged fermentation. Biotechnol. Bioeng., v. 57, p. 409-419, 1998.

JONCZYK, P.; TAKENBERG, M.; HARTWIG, S.; BEUTEL, S.; BERGER, R. G.; SCHEPER, T. Cultivation of shear stress sensitive microorganisms in disposable bag reactor systems. J. Biotechnol., v. 167, p. 370-376, 2013.

LINEK, V.; BENES, P.; SINKULE, J.; MOUCHA, T. Non-ideal pressure step method for $\mathrm{k}_{\mathrm{L}} \mathrm{a}$ measurement. Chem. Eng. Sci., v. 48, p. 1593-1599, 1993.

MARTINS, V. A. P.; LEENEN, E. J. T. M.; RIPPOLL, M. M.; VAN DER SLUIS, C.; VAN VLIET, T.; TRAMPER, J.; WIJFFLES, R.H. Relevance of rheological properties of gel beads for their mechanical stability in bioreactors. Biotechnol. Bioeng., v. 56, p. 517-529, 1997.

MERCHUK, J. C.; BEN-ZVI, S. (YONA). A novel transfer approach to the correlation of mass transfer rates in bubble columns with non- newtonian liquids. Chem. Eng. Sci., v. 47, p. 3517-3523, 1992.

NISHIKAWA, M.; KATO, H.; HASHIMOTO, K. Heat transfer in aerated tower filled with non-newtonian liquid. Ind. Eng. Chem. Process Des. Dev., v. 16, p. 133-137, 1977.

SCHUMPE, A.; DECKWER, W. D. Viscous media in tower bioreactors: Hydrodynamic characteristics and mass transfer properties. Bioprocess Eng., v. 2, p. 79-94, 1987. 